Introduction: Carcinosarcoma (CS) is an infrequent neoplasm composed of a carcinomatous and a sarcomatous element. Its molecular pathogenesis is poorly understood. In this study, we investigated the disturbances in the immunohistochemical expression of p53 and mismatch repair (MMR) proteins, as well as their molecular background.

Material and methods: The study group consisted of 20 uterine CSs. We analysed their morphology and immunohistochemical expression of hMLH1, hPMS2, hMSH2, MSH6, and p53 as well as the presence of mutations in TP53 and promoter methylation of the hMLH1. Loss of hMLH1 and PMS2 was found in 3/20 tumours. All cases were positive for hMSH2 and hMSH6. The TP53 mutation was detected in 8/19 tumours (42.1\%), whereas MLH1 promoter hypermethylation in $4 / 19$ cases (21\%), and one case with synchronous aberrations $(5 \%)$. Agreement between the results of the genetic and immunohistochemical study was moderate for $\mathrm{p} 53$ $(\kappa=0.615, p<0.01)$ and strong for MLH1 $(\kappa=0.826, p<0.01)$.

Results and conclusions: We demonstrated MLH1 promoter hypermethylation in uterine CS, leading to loss of MLH1 immunostaining. Concomitant aberrations of $\mathrm{p} 53$ and $\mathrm{hMLH} 1$ are infrequent. It is likely that uterine CS may develop in two independent molecular pathways in association with either chromosomal or microsatellite instability.

Key words: carcinosarcoma, mismatch repair proteins, p53, epithelial-mesenchymal transition, uterus.

Contemp Oncol (Pozn) 2019; 23 (4): 202-207 DOI: https://doi.org/10.5114/wo.2019.89635

\section{MLH1 promoter hypermethylation in uterine carcinosarcoma rarely coexists with TP53 mutation}

\author{
Michal Kunc ${ }^{1}$, Anna Gabrych ${ }^{2}$, Bartlomiej Rekawiecki ${ }^{3}$, Adam Gorczynski ${ }^{1}$, \\ Sabine Franke ${ }^{4}$, Johannes Haybaeck ${ }^{4,5,6}$, Wojciech Biernat ${ }^{1}$, Piotr Czapiewski ${ }^{1,4}$ \\ ${ }^{1}$ Department of Pathomorphology, Medical University of Gdansk, Poland \\ ${ }^{2}$ Department of Paediatrics, Haematology and Oncology, Medical University of Gdansk, \\ Gdansk, Poland \\ ${ }^{3}$ Department of Pulmonology, Medical University of Gdansk, Gdansk, Poland \\ ${ }^{4}$ Institute of Pathology, Otto-von-Guericke University Magdeburg, Germany \\ ${ }^{5}$ Institute of Pathology, Medical University of Graz, Austria \\ ${ }^{6}$ Department of Pathology, Neuropathology and Molecular Pathology, Medical University \\ of Innsbruck, Austria
}

\section{Introduction}

Carcinosarcomas (CSs) are rare tumours that consist of two malignant components: carcinomatous and sarcomatous, and are often associated with poor prognosis. These neoplasms have been observed in various locations, such as uterus, ovaries, breast, gastrointestinal tract, genitourinary tract, lung, breast, bones, thyroid gland, and paranasal sinuses [1-4]. The most common site of CS is the uterus, where it is referred to as mixed malignant Müllerian tumour (MMMT). The ambivalent nature of CSs raises questions about its origin. In the vast majority of tumours, a monoclonal origin of both components was proven [5]. Comparative genetic analyses performed on various CSs (e.g. CS of parotid gland, urinary bladder, and pharynx) revealed a large overlap of chromosomal aberrations in both tumour components [6, 7].

Most authors presume that the sarcomatous component is derived from carcinoma through a process called epithelial-mesenchymal transition (EMT) [5]. Physiologically, EMT is involved in the formation of the body plan during foetal development, differentiation of tissues, and their repair. Epithelial cells undergoing EMT lose the epithelial phenotype and acquire an increased migration potential. In various cancers, partial or complete EMT occurs, thus enhancing the malignant potential [8]. The hypothesis of the crucial role of EMT in CS pathogenesis is supported by ultrastructural, immunohistochemical, and microRNA studies [9-12].

TP53 mutations seem to be crucial for CS pathogenesis. In the vast majority of CSs, concordant overexpression of p53 is found in both components [13-15]. In recent years, molecular research on uterine CS has highlighted the role of p53 and mismatch repair protein (MMR) dysfunction in its pathogenesis; however, some studies produced conflicting data, and the role of $\mathrm{MLH1}$ promoter hypermethylation has not yet been evaluated in these tumours. Therefore, we investigated the p53 and MMR status in uterine CS obtained from a single institution cohort.

\section{Material and methods}

\section{Study population}

Cases of CS, recorded at the University Clinical Centre in Gdansk from 2007 to 2015, were retrieved from the archive of the Department of Pathomorphology. The microscopic slides of each case were re-evaluated by two pathologists to verify the diagnosis. Twenty formalin-fixed and paraffinembedded (FFPE) tissue blocks containing representative uterine CS samples were included in our study. 

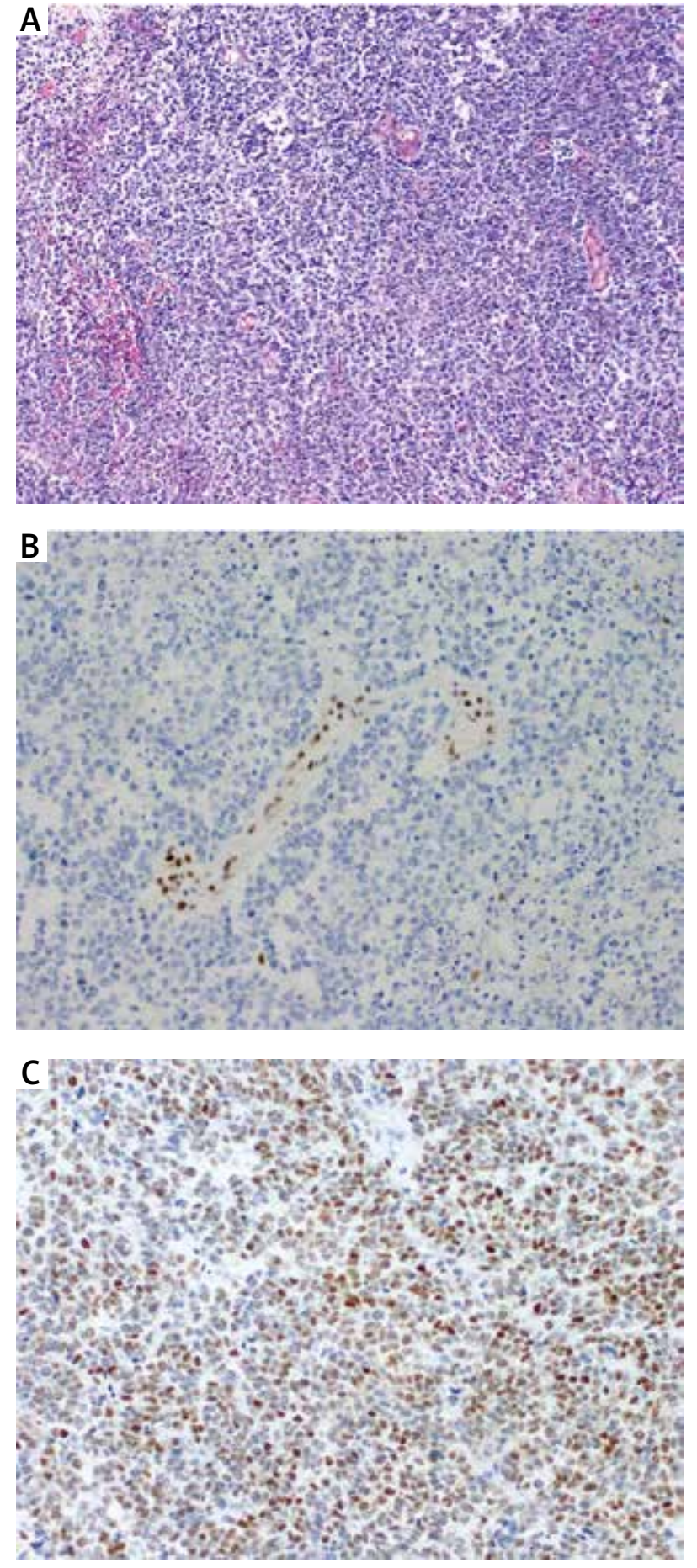

Fig. 1. Carcinosarcoma (H\&E) (A) with loss of MLH1 (B) and inhomogeneous p53 (C). MLH1 promoter hypermethylation, p53 wildtype

\section{Immunohistochemistry}

Tumour samples were stained with antibodies against hMLH1(Clone ES05), PMS2 (Clone EP51), hMSH2 (Clone FE 11), hMSH6 (Clone EP49), and p53 (Clone DO-7), all ready to use (DAKO, Denmark). Staining was performed on a Dako autostainer according to the manufacturer's instructions. The slides of all specimens were microscopically evaluated by two experienced pathologists (PC and WB). In the case of MMR proteins, nuclear staining was considered positive,
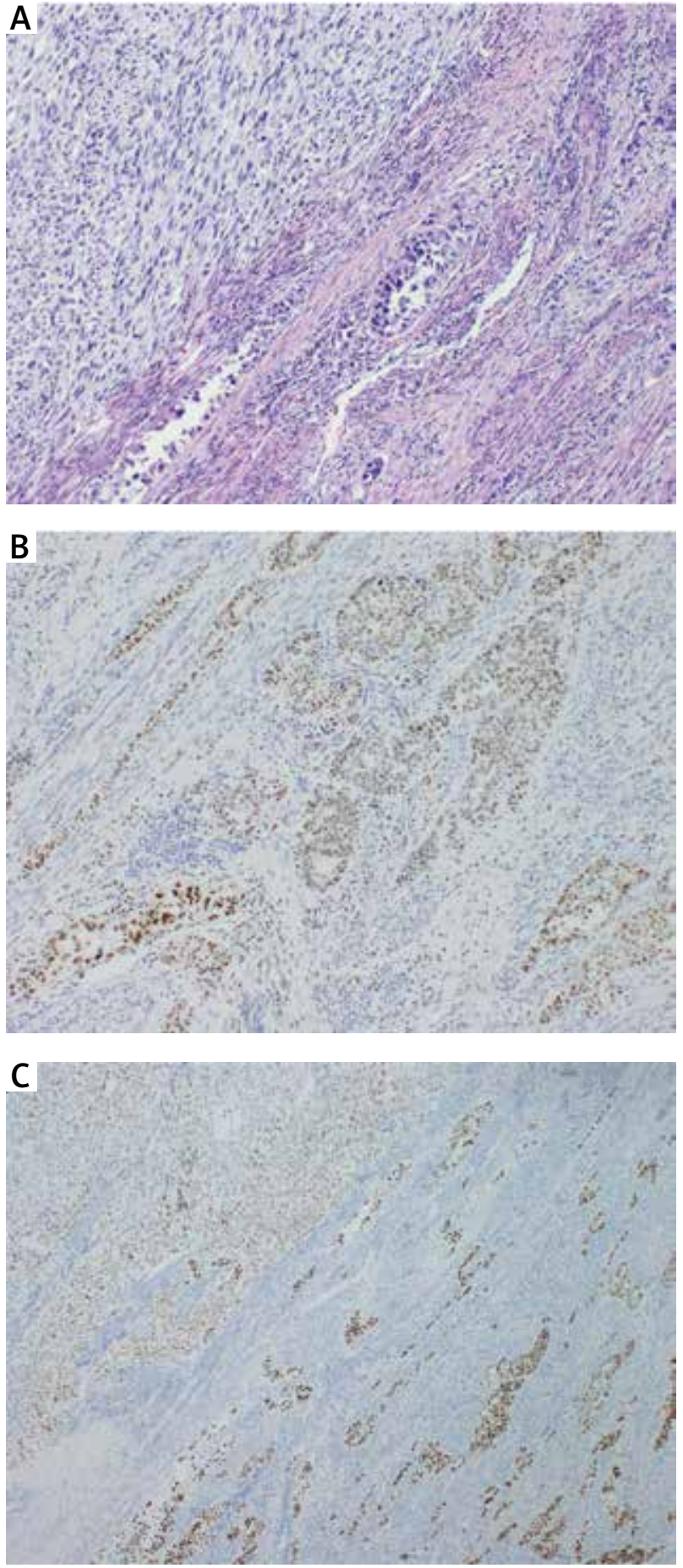

Fig. 2. Carcinosarcoma (H\&E) (A) with retained expression of $M L H 1$ (B) and strong expression of $\mathrm{p} 53$ (C). MLH1 promoter hypermethylation, p53 mutated

and lack of nuclear staining - negative (a sign of defective MMR). The question of how to correctly interpret the immunostaining for p53 is a matter of controversy. According to the current point of view, we considered a strong/diffuse (> 75\% of tumour cell nuclei) and a completely negative stain as p53 defect indicative of its mutation, (missense and nonsense, respectively), whereas a patchy/scattered pattern was regarded as a marker of normal p53 function [16]. Representative pictures are given in Figures 1 and 2. 


\section{DNA extraction from FFPE tissue sections}

Serial 6- $\mu \mathrm{m}$ slices were cut from the paraffin-embedded blocks, and the first section was stained with haematoxylin-eosin (HE) to evaluate the tumour cell content. If the tumour cell content was less than 50\%, macrodissection of tumour cell-rich areas was performed. After deparaffinisation, DNA was extracted according to the standard procedure described in the Cobas ${ }^{\oplus}$ DNA Sample Preparation Kit (Roche Molecular Systems, Inc., USA) package insert. The amount of genomic DNA was adjusted to fixed concentrations of $2-5 \mathrm{ng} / \mu \mathrm{l}$.

\section{TP53 mutation analysis}

TP53 mutation status in tissue samples was assessed by PCR using MyTaq HS DNA polymerase (Bioline, Germany) through 37 cycles with appropriate annealing temperatures. Primer sequences and annealing temperatures specific for exons 4-8 of TP53 are shown in Table 1. PCR samples were subjected to direct sequencing of single-stranded PCR products using a BigDye ${ }^{\oplus}$ Terminator v1.1 cycle sequencing kit and an ABI 3500 genetic analyser (Applied Biosystems).

\section{MLH1 methylation analysis}

Bisulphite conversion of genomic DNA from tissue samples was performed using the InviGene ${ }^{\oplus}$ Bisulfite Conversion Kit (STRATEC Molecular $\mathrm{GmbH}$ ) according to the manufacturer's instructions. MLH1 promoter methylation status was assessed by comparing normal genomic DNA with bisulphite-treated DNA of each tissue sample using methylated and unmethylated allele-specific primers and PCR conditions as described above. Primer sequences and annealing temperatures are shown in Table 1. PCR samples were subjected to an Agilent 2100 Bioanalyzer using the Agilent DNA 1000 Kit according to the manufacturer's instructions (Agilent Technologies).

\section{Statistical analysis}

Concordance in immunohistochemical staining between sarcomatous and carcinomatous components was evaluated with Fisher's exact test and kappa test. Other categorical variables were compared using Fisher's exact test. Correlation between MMR and p53 status was assessed by Spearman's rank-correlation test. Calculations were performed using Statistica software (Dell, version 13), licensed to the Medical University of Gdansk.

\section{Results}

Twenty patients were included in the study. Immunohistochemical staining patterns of each tumour, along with basic demographic data, are shown in Table 2. Median age at diagnosis was 71.5 years (range 54 to 88, average 72.5). All cases occurred in post-menopausal patients. Immunostaining for hMLH1 and PMS2 were negative in three out of 20 uterine CS (15\%). In turn, immunohistochemically, hMSH2 and hMSH6 were present in all tumours. There was a concordance rate of $100 \%$ in MMR protein staining between both components $(\kappa=1, p<0.001)$.

We detected abnormal IHC expression of p53 in 12 of 20 samples $(60 \%)$. In the vast majority of cases $(18 / 20$, $90.0 \%$ ) there was concordance between the sarcomatoid and carcinomatous components $(\kappa=0.895, p<0.001)$. Aberrant p53 staining was strong/diffuse in all cases.

Interestingly, we observed that in all three cases with defective MMR, there was no immunohistochemical evidence of any p53 defect; on the other hand, all uterine tumours with defective $\mathrm{p} 53$ were positive for MMR proteins. Thus, in the next step, we tested the MLH1 promoter methylation status and TP53 mutations in our cohort (one case was excluded due to the lack of representative material for further analyses). TP53 mutation was detected in 8/19 tumours (42.1\%), whereas MLH1 promoter hypermethylation occurred in 4/19 cases (21\%). Only one case showed a simultaneous defect in TP53 and hMLH1 (5\%). TP53 mutations were found exclusively in regions responsible for DNA binding, and some of them had been previously observed in uterine CS. Agreement between the results of the genetic and immunohistochemical study was moderate for p53 $(\kappa=0.615, p<0.01)$ and strong for hMLH1 $(\kappa=0.826, p<0.01)$. Correlation between p53 and hMLH1 defect in uterine CS, assessed by Spearman's rank-correlation, was $-0.40849(p=0.0825)$.

\section{Discussion}

We hypothesised that loss of mismatch repair (MMR) proteins and p53 expression, caused by genome instability and generation of either microsatellite (MSI) or chromosomal instability (CIN), may play a role in the pathogenesis of uterine CS. It is well known that MMR-deficiency and TP53 mutations, either gain or loss of function, may lead to the development of the sarcomatous component, for example through TGF- $\beta$ signalling (by MMR deficiency) [17] or miRNA $[18,19]$.

Table 1. Primer sequences specific for TP53 as well as methylated (met) and unmethylated (unmet) MLH1 promoter

\begin{tabular}{lccc} 
Primer & \multicolumn{1}{c}{ Forward } & Reverse & Temperature \\
p53 exon 4 & ATCTACAGTCCCCCTTGCCG & GCAACTGACCGTGCAAGTCA & $60^{\circ} \mathrm{C}$ \\
p53 exon 5 & TTCACTTGTGCCCTGACTT' & ACCCTGGGCAACCAGCCCTGTC & $60^{\circ} \mathrm{C}$ \\
p53 exon 6 & ACCATGAGCGCTGCTCAGAT & AGTTGCAAACCAGACCTCAGGC & $60^{\circ} \mathrm{C}$ \\
p53 exon 7 & CTGCTTGCCACAGGTCTC & TGGATGGGTAGTAGTATGGAAG & $60^{\circ} \mathrm{C}$ \\
p53 exon 8 & ACTGCCTCTTGCTTCTCTTT & AAGTGAATCTGAGGCATAAC & $56^{\circ} \mathrm{C}$ \\
MLH1 unmet & GAAGAGTGGATAGTGATTTTTAATGT & ATCTCTTCATCCCTCCCTAAACA & $60^{\circ} \mathrm{C}$ \\
MLH1 met & AGCGGATAGCGATTTTTAACGC & TCTTCGTCCCTCCCTAAAACG & $60^{\circ} \mathrm{C}$
\end{tabular}



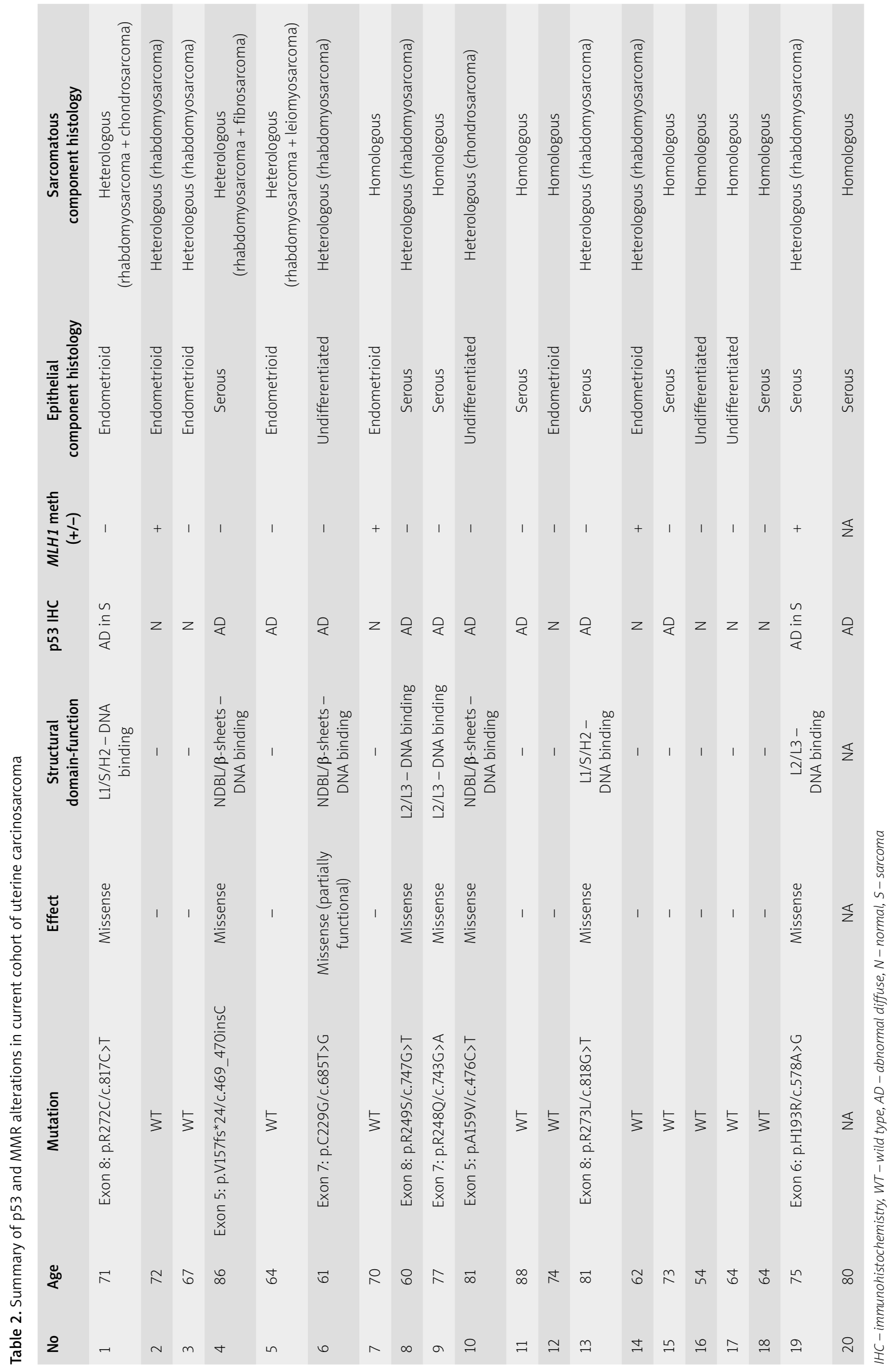
In previous studies, conflicting results have been published. In their prototypical study, Taylor et al. examined 28 uterine CS for MSI and MMR protein (hMLH1, hMSH2, and hMSH6) expression and TP53 status [20]. Six tumours (21\%) displayed defective DNA mismatch repair owing to the high-level MSI (MSI-H) phenotype in at least one component. Interestingly, immunostaining was negative for hMLH1, hMSH2, and hMSH6 [20] in only two of six cases with MSI-H. Other studies reported IHC MMR protein loss in CSs at various frequencies, ranging from $3 \%$ to $40 \%$ [21-23]. Only one study reported frequent IHC loss of hMSH2 and hMSH6 in CS [23]. However, the results of the IHC studies of MMR proteins without molecular confirmation should be interpreted with caution. The MSI frequency in CS of the female genital tract ranges from $5 \%$ to $23 \%$ of cases $[20-22,24,25]$. Overall, the frequency of MMR defects assessed with the use of various methods reported in the literature is $13 \%$ [26]. The two main mechanisms of MMR loss in cancer are sequence mutations and epigenetic changes. Extensive parallel sequencing of 22 uterine CSs revealed $M L H 1$ frameshift mutation in one case (5\%) and MSH6 nonsense mutation in three cases (15\%) [27]. A recent comprehensive epigenetic analysis of 57 uterine CS cases revealed $M L H 1$ epigenetic silencing in two tumours exhibiting MSI, discovering a novel important mechanism responsible for MMR deficiency in uterine CS [28]. Our study reports another four cases of MLH1 promoter methylation in CS, which supports these findings.

We have observed MMR defect in 15\% of cases according to the $\mathrm{IHC}$ and $21 \%$ according to the $\mathrm{MLH} 1$ promotor hypermethylation analysis. This discrepancy was shown in a previous study from Australia; in 702 endometrial cancer patients it was demonstrated that 3\% of MMR-proficient tumours showed MLH1 promoter methylation, and the methylation levels in these cases were low [29].

Taylor's cohort and ours showed a tendency towards an inverse association between MMR and p53 status [20]. Only one tumour from our cases presented with MLH1 hypermethylation and TP53 mutation; however, it was still positive immunohistochemically. Moreover, in our cohort, MLH1 promoter hypermethylation occurred preferentially in cases with the endometrioid epithelial component, whereas p53 aberrations were seen in CS with the serous or undifferentiated component. These findings are consistent with another study that demonstrated a higher frequency of p53 defects in CS with serous differentiation when compared with CS with an endometrioid epithelial component [30]. Genetic studies showed that the overall mutation profile of uterine CS corresponds to the mutation profiles of either serous (mutation of TP53 and PPP2R1A) or endometrioid carcinomas (mutation of PTEN and ARID1A) [21, 31]. Nevertheless, these findings are not supported by other studies that failed to demonstrate any inverse associations between mutations in MMR genes and TP53 [21, 28].

\section{Conclusions}

We conclude that epigenetic loss of hMLH1 expression may be an element of pathogenesis in some cases of uterine CS. In our cohort, the MMR defect tended to show an inverse correlation with p53 abnormalities. This association may indicate the existence of two distinct pathways of uterine CS development: through microsatellite or chromosomal instability. As CSs are rare tumours, multicentre studies are required to better explain the role of these alternations in tumours originating from the uterus and other organs.

The authors declare no conflict of interest.

\section{References}

1. Völker HU, Mühlmeier G, Maier H, Kraft K, Müller-Hermelink HK, Zettl A. True malignant mixed tumour (carcinosarcoma) of submandibular gland - a rare neoplasm of monoclonal origin? Histopathology 2007; 50: 795-798.

2. Okamura J, Sekine S, Nara S, Ojima H, Shimada K, Kanai Y, Hiraoka N. Intraductal carcinosarcoma with a heterologous mesenchymal component originating in intraductal papillary-mucinous carcinoma (IPMC) of the pancreas with both carcinoma and osteosarcoma cells arising from IPMC cells. J Clin Pathol 2010; 63: 266-269.

3. Nakagawa S, Nishimaki T, Suzuki T, Yokoyama N, Kuwabara S, Hatakeyama K. Histogenetic heterogeneity in carcinosarcoma of the esophagus: report of a case with immunohistochemical and molecular analyses. Dig Dis Sci 1999; 44: 905-909.

4. Blaukovitsch M, Halbwedl I, Kothmaier H, Gogg-Kammerer M, Popper HH. Sarcomatoid carcinomas of the lung - are these histogenetically heterogeneous tumors? Virchows Arch 2006; 449: 455-461.

5. McCluggage WG. Malignant biphasic uterine tumours: carcinosarcomas or metaplastic carcinomas? J Clin Pathol 2002; 55: 321-325.

6. Vékony H, Leemans CR, Ylstra B, Meijer GA, van der Waal I, Bloemena E. Salivary gland carcinosarcoma: oligonucleotide array $\mathrm{CGH}$ reveals similar genomic profiles in epithelial and mesenchymal components. Oral Oncol 2009; 45: 259-265.

7. Torenbeek R, Hermsen MA, Meijer GA, Baak JP, Meijer CJ. Analysis by comparative genomic hybridization of epithelial and spindle cell components in sarcomatoid carcinoma and carcinosarcoma: histogenetic aspects. J Pathol 1999; 189: 338-343.

8. Thiery JP, Acloque H, Huang RYJ, Nieto MA. Epithelial-mesenchymal transitions in development and disease. Cell 2009; 139: 871890.

9. Geisinger KR, Dabbs DJ, Marshall RB. Malignant mixed müllerian tumors An ultrastructural and immunohistochemical analysis with histogenetic considerations. Cancer 1987; 59: 1781-1790.

10. Sreenan JJ, Hart WR. Carcinosarcomas of the female genital tract A pathologic study of 29 metastatic tumors: further evidence for the dominant role of the epithelial component and the conversion theory of histogenesis. Am J Surg Pathol 1995; 19: 666-674.

11. Emoto M, Iwasaki H, Kikuchi M, Shirakawa K. Characteristics of cloned cells of mixed müllerian tumor of the human uterus carcinoma cells showing myogenic differentiation in vitro. Cancer 1993; 71: 3065-3075.

12. Castilla MÁ, Moreno-Bueno G, Romero-Pérez L, et al. Micro-RNA signature of the epithelial-mesenchymal transition in endometrial carcinosarcoma. J Pathol 2011; 223: 72-80.

13. Armstrong AB, Wang M, Eble JN, et al. TP53 mutational analysis supports monoclonal origin of biphasic sarcomatoid urothelial carcinoma (carcinosarcoma) of the urinary bladder. Mod Pathol 2009; 22: 113-118.

14. Amatya VJ, Takeshima Y, Kaneko M, Inai K. Esophageal carcinosarcoma with basaloid squamous carcinoma and rhabdomyosarcoma components with TP53 mutation. Pathol Int 2004; 54: 803-809.

15. Keeling L, Taraporewalla D, Perunovic B, Smith JHF. Uterine carcinosarcoma with p53-positive intraepithelial component. Histopathology 2011; 59: 1277-1278.

16. Murali R, Davidson B, Fadare O, et al. High-grade Endometrial Carcinomas. Int J Gynecol Pathol 2019; 38: S40-S63. 
17. Dwivedi SKD, McMeekin SD, Slaughter K, Bhattacharya R. Role of TGF- $\beta$ signaling in uterine carcinosarcoma. Oncotarget 2015; 6: 14646-14655.

18. Dong P, Karaayvaz M, Jia N, et al. Mutant p53 gain-of-function induces epithelial-mesenchymal transition through modulation of the miR-130b-ZEB1 axis. Oncogene 2013; 32: 3286-3295.

19. Chang C-J, Chao C-H, Xia W, et al. p53 regulates epithelial-mesenchymal transition and stem cell properties through modulating miRNAs. Nat Cell Biol 2011; 13: 317-323.

20. Taylor NP, Zighelboim I, Huettner PC, et al. DNA mismatch repair and TP53 defects are early events in uterine carcinosarcoma tumorigenesis, Mod. Pathol 2006; 19: 1333-1338.

21. McConechy MK, Hoang LN, Chui MH, et al. In-depth molecular profiling of the biphasic components of uterine carcinosarcomas. J Pathol Clin Res 2015; 1: 173-185.

22. Taylor NP, Gibb RK, Powell MA, Mutch DG, Huettner PC, Goodfellow PJ. Defective DNA mismatch repair and XRCC2 mutation in uterine carcinosarcomas. Gynecol Oncol 2006; 100: 107-110.

23. de Jong RA, Nijman HW, Wijbrandi TF, Reyners AK, Boezen HM, Hollema $\mathrm{H}$. Molecular markers and clinical behavior of uterine carcinosarcomas: focus on the epithelial tumor component. Mod Pathol 2011; 24: 1368-1379.

24. Fujii H, Yoshida M, Gong ZX, et al. Frequent genetic heterogeneity in the clonal evolution of gynecological carcinosarcoma and its influence on phenotypic diversity. Cancer Res 2000; 60: 114-120.

25. Amant F, Dorfling CM, Dreyer L, Vergote I, Lindeque BG, Van Rensburg EJ. Microsatellite instability in uterine sarcomas. Int J Gynecol Cancer 2001; 11: 218-223.

26. Hoang LN, Ali RH, Lau S, Gilks CB, Lee C-H. Immunohistochemical survey of mismatch repair protein expression in uterine sarcomas and carcinosarcomas. Int J Gynecol Pathol 2014; 33: 483-491.

27. Jones S, Stransky N, McCord CL, et al. Genomic analyses of gynaecologic carcinosarcomas reveal frequent mutations in chromatin remodelling genes. Nat Commun 2014; 5: 5006.

28. Cherniack AD, Shen H, Walter V, et al. Integrated Molecular Characterization of Uterine Carcinosarcoma. Cancer Cell 2017; 31: 411-423.

29. Buchanan DD, Tan YY, Walsh MD, et al. Tumor mismatch repair immunohistochemistry and DNA MLH1 methylation testing of patients with endometrial cancer diagnosed at age younger than 60 years optimizes triage for population-level germline mismatch repair gene mutation testing. J Clin Oncol 2014; 32: 90-100.

30. Lopez-Garcia M-A, Palacios J. Pathologic and molecular features of uterine carcinosarcomas. Semin Diagn Pathol 2010; 27: 274-286.

31. Zhao S, Bellone S, Lopez S, et al. Mutational landscape of uterine and ovarian carcinosarcomas implicates histone genes in epithelial-mesenchymal transition. Proc Natl Acad Sci U S A 2016; 113: 12238-12243.

\section{Address for correspondence}

\section{Piotr Czapiewski}

Institute of Pathology

Otto-von-Guericke University Magdeburg

Leipziger Str. 44

39108 Magdeburg, Germany

e-mail: piotr.czapiewski@med.ovgu.de

Submitted: 17.10 .2019

Accepted: 24.10 .2019 